\title{
Las agendas internacionales y el desarrollo urbano local. Una revisión por los modelos de planificación e instrumentos adoptados por la gestión urbana local en Córdoba, Argentina (1983-2019) ${ }^{1}$
}

\section{International agendas and local urban development. A review of planning models and instruments adopted by local urban management in Córdoba city, Argentina (1983-2019).}

\author{
Natalí Peresini² ${ }^{\circledR 0}$
}

\begin{abstract}
RESUMEN
La ciudad latinoamericana, sin pretender generalizar sus particularidades, en los últimos años ha guiado su proceso de urbanización principalmente bajo el paradigma de "desarrollo urbano". Así también, en la región se han adoptado modelos de planificación urbana similares en ciudades con realidades y coyunturas disimiles. Ante ello, nos proponemos revisar los principales conceptos y modelos difundidos en las últimas cuatro décadas por organismos internacionales que han tenido impacto en la orientación de la gestión, políticas y planificación urbana local al mismo tiempo que se identifica cómo han ido evolucionando y qué incidencia han alcanzado al rescatar aquellos efectivamente implementados en un caso de estudio. Para cumplir con el objetivo se adoptó una estrategia metodológica de base cualitativa que alterna el análisis de documentos con la indagación empírica en la ciudad de Córdoba (Argentina) en el período 1983-2019. Los resultados arrojan una progresiva incidencia tanto de los paradigmas de planificación como de los modelos de desarrollo propuestos por los organismos internacionales en la gestión urbana local, exponiendo mayor influencia a partir de los años `90. Así también la adaptación paulatina pero continua de la planificación urbana hacia modalidades neoliberales que aseguren injerencia y capacidad de acción a los intereses y actores empresariales.
\end{abstract}

Palabras claves: Desarrollo urbano, Planificación urbana, Gestión urbana local, Urbanismo neoliberal.

Este artículo recupera parte de los resultados producidos en el marco del proyecto de tesis doctoral que se desarrolla en el programa de Estudios Urbanos de la Universidad Nacional de General Sarmiento (Bs. As., Argentina) y gracias al financiamiento del Consejo Nacional de Investigaciones Científicas y Técnicas. Se agradece a Joaquín Peralta por la información y los documentos que nutren este artículo. Becaria doctoral. Consejo Nacional de Investigaciones Científicas y Técnicas -CONICET- Correo electrónico: natperesini@gmail.com 


\begin{abstract}
The Latin American city, without generalize, in recent years has guided its urbanization process under the paradigm of "urban development". Thus also, similar urban planning models have been adopted in cities with dissimilar realities and conjunctures in the region. We propose review the main concepts and models disseminated in the last four decades by international organizations. Focusing in that concepts wich have had an impact on the direction of management, policies and local urban planning. Also identifying how they have evolved and what impact they have had rescuing those actually implemented in a case study. To fulfill this objective, a qualitatively based methodological strategy was adopted. We altarate the revision and analysis of documents with empirical research in the Córdoba city (Argentina) in the period 1983-2019. The results show a progresive and mayor impact of the planning paradigms and the development models proposed by the international organizations on local urban management since 1990. As well the gradual but continuous adaptation of urban planning towards neoliberal modalities that ensure interference and capacity for action to business interests and actors.
\end{abstract}

Keywords: Urban development, urban planning, Local urban management, Neoliberal urbanism.

Existe un consenso generalizado que define que, desde la posguerra y hasta la década del ' 70 , la atención gubernamental en relación a lo urbano se concentró fundamentalmente en la mejora de las condiciones de vivienda y habitabilidad con protagonismo y responsabilidad casi exclusiva del sector estatal (Ballent, 2005; Puebla Cadena, 2002; Pradilla Cobos, 2009). Así también, se reconoce que desde los años '90 toma fuerza un enfoque ligado a la noción de gobernanza, proponiendo un nuevo modo de gestionar las políticas urbanas que incluye la articulación de los recursos y capacidades estatales en respaldo y para facilitar las acciones empresariales (Harvey, 2007; Pradilla Cobos, 2012). En este giro de responsabilidades, capacidades e incumbencias, una serie de paradigmas y modelos de planificación urbana obtienen particular protagonismo, consiguiendo respaldo y difusión por parte de organismos internacionales y siendo adoptados e implementados en diferentes ciudades de la región, a pesar de tener realidades y coyunturas diversas.

Junto con ello, en Latinoamérica y fundamentalmente desde los años '90, el proceso de globalización, competitividad y apertura de mercados comerciales y financieros ha repercutido con especial énfasis en los gobiernos locales. Este proceso, en su evolución, ha generado complejos y profundos cambios en la organización socio espacial de las ciudades que revelan un interés particular del orden político-económico vigente por el proceso de urbanización contemporáneo (De Mattos, 2007; Pradilla Cobos, 1990).

Siguiendo los aportes de los estudios urbanos críticos, las ciudades se han convertido en las últimas décadas en un ámbito estratégico para los proyectos de neoliberalización, ya que sirven como campo de incubación, experimentación, despliegue y avance de políticas estatales y restructuraciones institucionales hacia modalidades neoliberales en sus múltiples dimensiones (Peck et al., 2009). Los autores sostienen que se ha producido una profundización de lo que llaman "urbanismo neoliberal", definido como una orientación de las políticas urbanas que promueven la mercantilización y especulación en cada componente de la ciudad, entendida como objeto de negocio.

En este marco, nos preguntamos entonces cuáles han sido los conceptos y modelos difundidos en los últimos años por entidades internacionales que han logrado impactar en la gestión, las 
políticas y la planificación urbana a escala local y con ello cuál o cuáles han sido las orientaciones promovidas. El presente artículo realiza una revisión diacrónica que procura identificar los paradigmas, modelos y conceptos promovidos y su evolución, así como reconocer qué tendencia han provocado a lo largo de las últimas cuatro décadas en las prácticas e instrumentos implementados por la gestión urbana local. Atendiendo que es un proceso en curso y no acabado, inferimos que hasta ahora la orientación e influencia de los modelos y paradigmas difundidos por los organismos más destacados en el campo de la planificación y la gestión urbana han colaborado en la consolidación del proceso de neoliberalización en la escala local (Brenner \& Theodore, 2002; Peck et al., 2009). Sostenemos que han acompañado de manera coherente y consistente al proceso de neoliberalización del urbanismo mediante, entre otras acciones, fomentar una serie de cambios en la interpretación de la planificación, los objetivos y alcances de las políticas urbanas a escala local.

\section{El "desarrollo urbano" como eje de interés de los organismos interna- cionales}

En 1972 el Banco Mundial (BM) presenta su primer programa considerando los problemas urbanos en los "países en desarrollo" (Van Lindert, 2016). En el mismo, se ponía en cuestión el uso de la planificación tecnocrática y los modelos urbanos utilizados en base al Estado de Bienestar que habian predominado hasta la fecha. Desde este primer informe, el BM se posicionó en el ámbito latinoamericano como una institución legitimada para recomendar, ofrecer líneas de acción y orientar las políticas urbanas (Zanetta, 2001).

Ahora bien, podemos preguntarnos qué interés puede tener una organización financiera internacional en la planificación de las ciudades. Con perspectiva histórica, el BM nació en la década del cuarenta para acompañar la reconstrucción de las ciudades en la post guerra, evolucionando hasta la actualidad como un organismo de crédito para "países en desarrollo". En Latinoamérica es acompañado por el Fondo Monetario Internacional y el Banco Interamericano de Desarrollo (BID), instituciones que también tienen entre sus funciones ser fuente de financiamiento para los países de la región. En la actualidad todas ellas otorgan préstamos para desarrollar proyectos "modernizadores" que se encuentren en sus programas y, con ello, incidir y condicionar las políticas, mecanismos y acciones en las ciudades latinoamericanas que adquieren dicho financiamiento.

Así también, desde la década del setenta otras instituciones como la Organización de las Naciones Unidas (ONU) a través de ONU-Hábitat o la Comisión Económica para América Latina y el Caribe (CEPAL), entre otras, han difundido de manera periódica y continuada una serie de recomendaciones destinadas a "promover el desarrollo económico y social de la región" ${ }^{3}$ enfocadas particularmente en el "desarrollo urbano".

En el devenir de los programas y publicaciones de estos organismos podemos identificar cambios en los objetivos o prioridades que se intentan divulgar y fomentar, sin embargo, encontramos una continuidad: el "desarrollo urbano" (DU) se presenta como propósito ineludible de las políticas y la gestión urbana. Un polisémico y a la vez poco cuestionado paradigma que es ampliamente utilizado en la gestión urbana para justificar planes y proyectos y que se ha configurado como el eje vector de las ciudades latinoamericanas en las últimas décadas (De Mattos, 2007; Gaspar, 
2008; Van Lindert, 2016). Su interpretación tanto conceptual como pragmática, consiste en vincular la idea de progreso al crecimiento de la actividad económica urbano-inmobiliaria, a la vez que pregona intensificar la relación entre los actores empresariales y gubernamentales.

\section{Marco metodológico}

Este artículo recupera parte de los avances y resultados producidos en tesis doctoral, la cual se enmarca en una estrategia metodológica de base cualitativa. Se propone una indagación relacional y diacrónica mediante una lectura que alterna el análisis de documentos de organismos internacionales en relación a la difusión de paradigmas, modelos y prácticas de planificación urbana y su adopción realmente existente en un gobierno local a través de un estudio de caso como método de aproximación.

Respecto del caso seleccionado, se hace foco en la gestión y planificación urbana implementada entre 1983-2019 en la ciudad de Córdoba, Argentina. Una de las primeras ciudades del país y de la región en contar con un plan urbano regulador en la primera mitad del siglo XX además de sumar en su trayectoria una larga lista de antecedentes en este campo, entre intentos y proyectos formales de planificación urbana (Colautti, 2013; Malecki, 2018; Caporossi, 2006).

Se abordan como dimensiones para el análisis, los paradigmas y/o modelos de planificación difundidos por los principales organismos internacionales y los modelos, mecanismos e instrumentos de gestión y planificación urbana adoptados en el caso de estudio. Como técnica de recolección y análisis de información se optó por la triangulación de datos ya que habilita el uso de diversas fuentes y métodos, permitiendo lograr una perspectiva más compleja en la interpretación del problema planteado. Para este trabajo se utilizó el cruce de tres herramientas, siendo:

- la indagación de fuentes y bibliografía relacionada;

- la recopilación y análisis de documentos oficiales (informes de organismos, normativas y planes urbanos del gobierno local) y,

- entrevistas semi estructuradas a actores claves.

En cuanto a las entrevistas se realizaron y sistematizaron una serie de diez entrevistas a personal técnico, funcionarios del gobierno local relacionados con el desarrollo urbano y referentes académicos. Los criterios de selección de los entrevistados se basaron en su identificación como informantes claves mediante la técnica bola de nieve y considerando su nivel de cercanía en la toma de decisiones respecto de la planificación y la gestión urbana que se analiza en esta investigación. Sumado a ello se realizaron algunos cruces con datos estadísticos de fuentes oficiales e instituciones académicas como fuentes complementarias.

El análisis se plantea en base a una periodización ad hoc de acuerdo a condiciones comunes para cada período, que comienza en 1983 dada la recuperación de la representación democrática en el municipio y en el país. Se intenta arrojar luz sobre qué modelos y mecanismos han sido implementados y qué impacto material e institucional han logrado en la escala local, siendo un nivel de gobierno que ha ocupado un rol preponderante en el proceso de globalización y competitividad trasnacional desde la década del ' 90 . 


\section{La ciudad de Córdoba, un caso de estudio}

Para abordar el caso de estudio haremos un breve repaso por algunos indiciadores socio-económicos que nos ayudan a contextualizar su coyuntura y situación. La ciudad de Córdoba es la segunda mayor poblada de la Argentina con 1.329.604 habitantes, de acuerdo a los datos del último censo oficial (INDEC, 2010), ha tenido un constante crecimiento de población desde su fundación (Figura $N^{\circ} 1$ ), aunque en los últimos registros se evidencia una tendencia decreciente. Su capacidad para retener habitantes ha ido en baja, afectada principalmente por la movilización de la población hacia ciudades aledañas (Tecco \& Valdés, 2006), sin embargo la mancha urbanizada se ha extendido ininterrumpidamente. Por su carácter de capital provincial concentra actividades de administración gubernamental, salud, educación provincial y nacional (universidades), actividad productiva industrial y de servicios, además de una red comercial consolidada. Los pilares de la economía lo constituyen el sector de servicios, comercio e industria, destacándose el rubro automotriz. Por todo ello, conforma la segunda mayor conurbación del país en una ubicación central en cuanto a su geografía y se constituye en un nodo articulador para el corredor comercial bioceánico y norte.

Su proceso de urbanización comienza marcado por una estrategia de ocupación territorial común a las ciudades latinoamericanas colonizadas, una cuadrícula de base y un modelo de organización y ordenamiento de acuerdo a las condiciones heredades. De dicha cuadrícula, se desprende la estructura física hasta la actualidad, conteniendo un centro histórico denso y consolidado; un anillo de barrios peri centrales de una primera expansión durante el siglo XIX; un área intermedia de baja densidad compuesta por barrios residenciales originados durante el proceso de industrialización a mediados del siglo XX; y, una periferia dispersa de muy baja densidad con características disímiles, ya que encontramos numerosos barrios cerrados destinados a grupos de alto nivel económico tanto como planes de vivienda social denominados "barrios ciudades" (plan de erradicación de villas financiado por el BID).

\section{Figura N01.}

Evolución de la mancha urbana y crecimiento de la población de la ciudad de Córdoba de acuerdo a fechas censales. 1810- 2010.

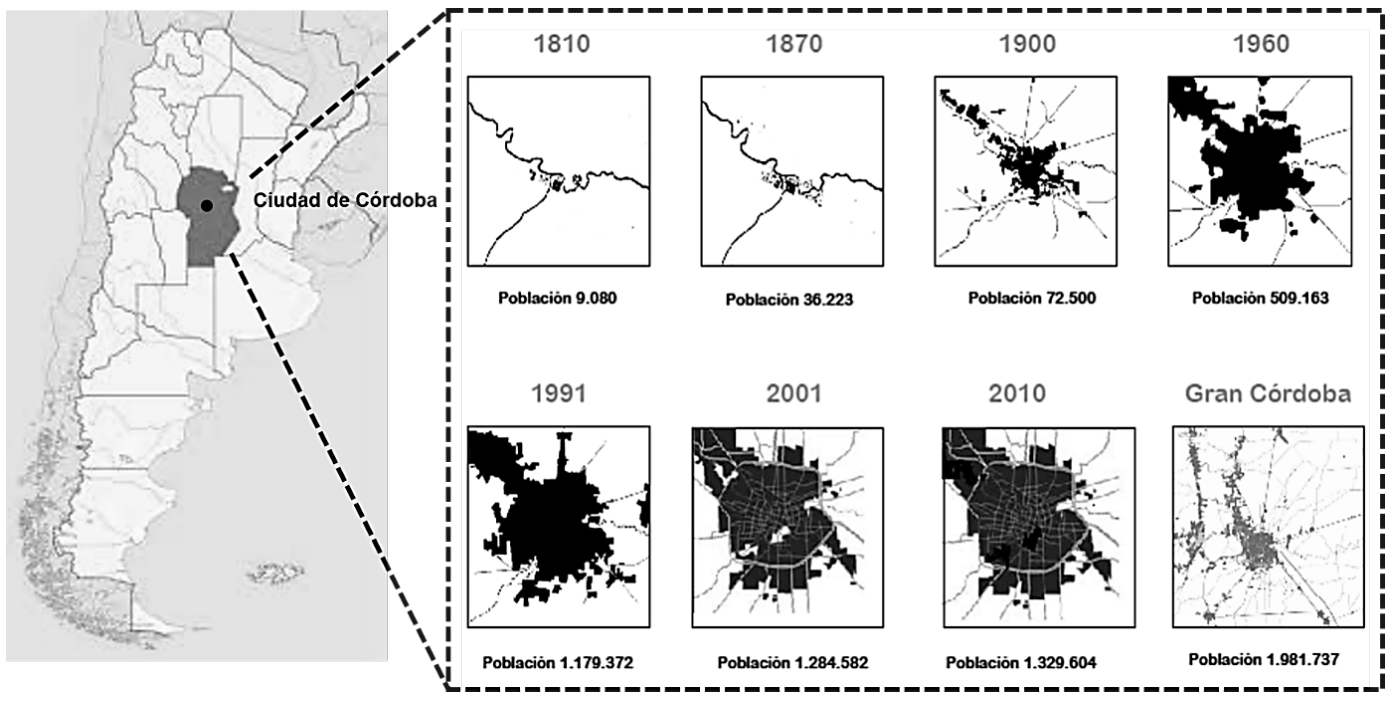

Fuente: elaboración propia en base a Municipalidad de Córdoba. 
El proceso de urbanización es acompañado, fundamentalmente en el último siglo, por un amplio historial de antecedentes en relación a la planificación urbana local que inicia con la configuración del plan regulador y de extensión para la ciudad realizado por Carrasco en 1920 (Stang, 2017) y otro por La Padula en 1962 (Malecki, 2018). Este último se convierte en un hito para la cultura y la memoria en el desarrollo y formación del urbanismo y la gestión urbana local que abona la producción de proyectos en este sentido y genera una alta valoración de la planificación urbana. En virtud de los numerosos antecedentes que se registran en este campo, se logra generar una genealogía de los modelos y la planificación elaborados y aplicados en el caso de estudio junto a las principales líneas de acción con el fin de comprender tanto la influencia como los efectos concretos y materiales de instrumentos y paradigmas hegemónicos impulsados durante las últimas cuatro décadas.

\section{Una revisión por los paradigmas y modelos de planificación urbana promovidos por los organismos internacionales y los adoptados en la ciudad de Córdoba (1983-2019)}

En este apartado, haremos un repaso por los dogmas fomentados desde los organismos internacionales y sus impactos en la política, gestión y planificación urbana local. Esta aproximación intenta resaltar la importancia de cuestionar quiénes construyen y promueven la agenda del desarrollo urbano, a quiénes benefician y cómo se implementan en un entorno material y contextualmente específico. En este sentido, destacamos una serie de eventos que se relacionan e inciden directamente en la agenda urbana latinoamericana y de los cuales se van a desprender las principales recomendaciones, modelos y paradigmas a la gestión urbana local. Ellos son: la primera Conferencia de Hábitat de la Organización de Naciones Unidas (ONU) en 1976, el Programa de Gestión Urbana de ONU-Hábitat y el Programa de las Naciones Unidas para el Desarrollo (PNUD) en 1986, la postulación de una agenda urbana ONU-Hábitat en 1991 y la Cumbre de la Ciudad en 1996 y, más recientemente, la secuencia de eventos denominado Foro urbano Mundial iniciado en 2002.

\section{La "agenda del desarrollo" en los ochenta: el Programa de Gestión Urbana de ONU-Hábitat y el PNUD}

En los años ochenta, tanto en el ámbito europeo como en el latinoamericano, se presentan algunas modificaciones en los paradigmas que regían la gestión de las ciudades. En el primer caso, se produce un cambio de posición del Estado respecto de su responsabilidad en solitario sobre las condiciones de habitabilidad, intentado establecer relaciones y compromisos compartidos con el sector privado y organizaciones sociales. En el escenario latinoamericano comienza un proceso de democratización, dejando atrás regímenes autoritarios en la mayoría de los países, en el cual las nuevas democracias necesitaban volver a organizar la administración pública (Van Lindert, 2016). En este marco se presenta un gran interés de las organizaciones internacionales para introducir en la agenda regional un cambio de enfoque, complejidad e incluso de escala en las políticas urbanas. Una variación importante es el salto que se produce en la interpretación y complejidad de la problemática, reemplazando la vivienda por la "cuestión urbana". Lo cual se 
plasma en varios documentos y propuestas a los fines de aplicar algunos puntos básicos de la "agenda del desarrollo" en los países de la región.

Específicamente en el campo del urbanismo latinoamericano ganan protagonismo los postulados propuestos en el Programa de Gestión Urbana de ONU-Hábitat y el PNUD lanzado en 1986, en los cuales tienden a equipararse las funciones de gobierno con las estrategias empresariales para la gestión de la ciudad. Paralelamente toma fuerza la noción de "nueva gestión pública" (New Public Managment o NPM) impulsada por académicos anglosajones (López, 2005), con el fin de incorporar herramientas empresariales en la gestión de gobierno. Durante esta década, se desarrolló principalmente un traslado de la lógica privada a las instituciones y funciones estatales para asegurar el "buen funcionamiento del mercado" y la buena administración pública bajo la consigna de eficientizar la gestión estatal.

La propia noción de gestión urbana para el manejo de las ciudades en la administración gubernamental surge en este contexto y bajo estos paradigmas, dejando de lado las prácticas que se ocupaban de ofrecer soluciones habitacionales o la planificación urbana como referencia para disciplinar, ordenar y contener el crecimiento de la ciudad para darle mayor consideración a aquellas acciones que van a promover la gestión por resultados (Van Lindert, 2016).

\section{La gestión urbana local: el desarrollo urbano y el modelo de zonning por valor del suelo (1983-1991)}

En este período en la ciudad de Córdoba, se refuerza la función de intervención y control estatal a escala local, impulsada también como estrategia para eficientizar la gestión pública. Respecto a la gestión urbana específicamente, se otorga un lugar de mayor jerarquía y poder de decisión al área encargada de ella, al ascender la dirección de planeamiento a secretaría de desarrollo urbano.

"El cambio no es menor, [...] supuso una jerarquía mayor en la toma de decisiones [...] Incluía por primera vez gestión, la planificación urbana ya no era solamente dibujar las manchas de uso del suelo, sino que era tomar decisiones de inversión. Esa era la gran diferencia entre planificación y desarrollo urbano" (J.P., entrevista, 24 de septiembre de 2018).

El término estrella de la época no sólo fue una denominación para el espacio institucional encargado del manejo de lo urbano, su control y planificación sino también para designar el plan general que se estableció por esos años. Bajo la premisa de ordenar y regular el crecimiento de la ciudad, acción legitimada históricamente en el campo disciplinar mediante instituciones estatales (Del Río et al., 2014), el Plan General de Desarrollo Urbano se concentró fundamentalmente sobre dos ejes: la legislación urbana y la obra pública (Iros, 1991). Al mismo tiempo se genera un programa de gestión ligado a los principios del DU como objetivo fundamental (Iros, 1991).

Para ello se estableció un modelo físico (Figura º2) que definió y diferenció áreas posibles de urbanizar, usos y densidades habilitadas para el aprovechamiento del máximo potencial de la infraestructura instalada y limitar al avance de suelo urbano sobre áreas rurales. Una propuesta con base en el funcionalismo y el criterio de "zonning", imitando la legislación moderna ya instalada en países europeos, concentrándose en definir áreas y usos -principio fundamental del urbanismo 
Figura $\mathrm{N}^{\circ} 2$.

Síntesis de la estructura urbana de la ciudad de Córdoba según PGDU. Córdoba, 1991.

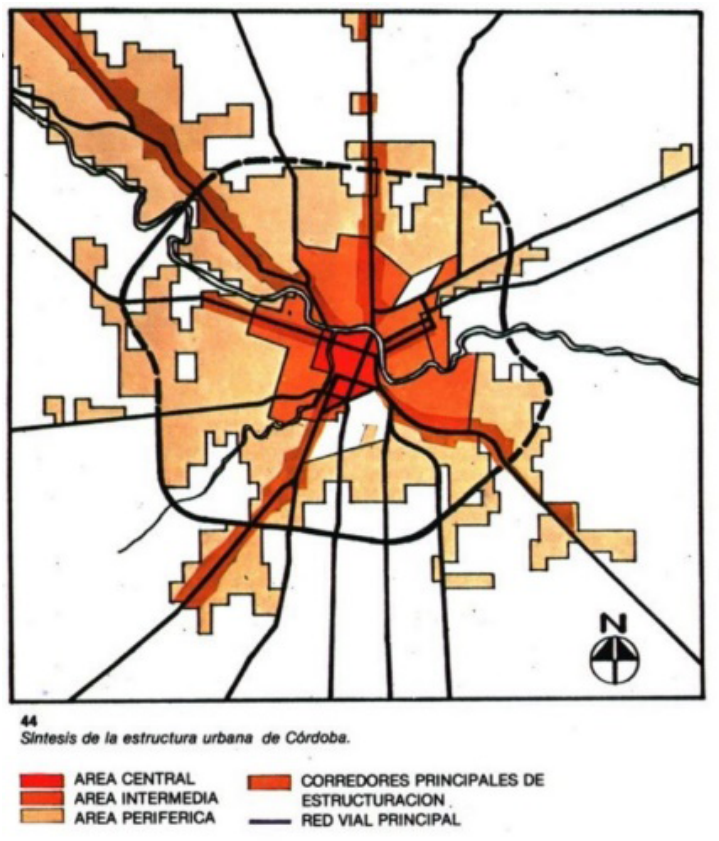

Fuente: Guillermo Iros (1991). "Desarrollo urbano y reflexiones".

moderno- a la vez que se recurre al paradigma de eficiencia para justificar la colaboración del sector privado para las inversiones productivas.

El esquema normativo zonifica en base a la renta del suelo urbano y establece tres reglas básicas que van a definir su rentabilidad: fraccionamiento (Ord. N8060), uso (Ord. N8133) y ocupación (Ord. N²8256/1986 áreas intermedias y periféricas y la Ord. №8057-área central). Al mismo tiempo, la zonificación y el marco regulatorio propuesto arrastra de planes anteriores la organización en anillos concéntricos al diferenciar la normativa según área central (núcleo histórico); intermedia (barrios peri centrales) y periférica. Este marco normativo, aún con numerosos cambios, sigue vigente hasta la actualidad y se configura como el principal dispositivo de homogenización y diferenciación de la renta de suelo urbano al definir limitaciones diferenciales sobre las propiedades de acuerdo a su localización, incidiendo en los precios y provocando una distribución particular de la renta del suelo urbano.

\section{Globalización, competitividad y gobernanza en los noventa: la Nueva Agenda Urbana y la Cumbre de la ciudad}

El modelo globalizador, de apertura de mercados comerciales y financieros iniciado durante los años noventa marcó una bisagra en las competencias y responsabilidades de los gobiernos locales. En el contexto regional la promulgación de leyes de descentralización administrativa fue esencial para acompañar este proceso. La desvinculación de la escala nacional de las problemáti- 
cas regionales y locales se fundamentó en la cercanía de los niveles subnacionales con las poblaciones afectadas, como también en la eficiencia para el uso de los recursos al ajustar las políticas al territorio (Aghón et al., 2001; Cao \& Vaca 2007).

Sumado a ello, la incorporación del enfoque gerencial promovido años anteriores sirvió de base para habilitar las prácticas descentralizadoras bajo los preceptos de que se construiría un Estado más abierto y eficaz, en consonancia con las ideas del "buen gobierno" (Cao et al., 2016). Apoyados en el principio de subsidiaridad, se efectuó la transferencia de funciones y servicios hacia los niveles subnacionales, aunque no de la misma forma los recursos para sostenerlos; lo que promovió la competitividad interurbana ante la obligación de generar y aumentar los recursos económicos-financieros en cada municipio.

En este contexto, la Nueva Agenda Urbana (NAU) presenta algunas estrategias para mejorar la competitividad de las ciudades. En el evento realizado en 1991 se hizo énfasis en el nuevo rol que debían atender, al destacar entre sus incumbencias y responsabilidades la captura "creativa e inteligente" de capitales o en caso contrario prepararse para que otras ciudades con mejor capacidad para promover el desarrollo económico lo hagan (Delgadillo, 2014).

Otro de los hitos que marcó esta década fue la masiva aceptación de la noción de gobernanza urbana. La incorporación del paradigma en la gestión local fue fundamental, tanto para los cambios en las responsabilidades y roles del gobierno municipal, como para legitimar la participación de actores privados. La difusión de esta modalidad de gobierno estuvo principalmente a cargo de organismos de crédito internacional (como el BM y el BID), acompañados por referentes específicos que tenían como objetivo impulsar y apoyar al poder local como escala de acción estratégica (Ferreira, 2001). Este enfoque venía acompañado por la idea de "buen gobierno" fomentado en la Cumbre de la ciudad en Hábitat II en 1996 (Van Lindert, 2016). Dicha conjunción implicaba centrar el papel de los municipios como un actor más, junto a la sociedad civil y los actores privados, teniendo como principal tarea estimular el desarrollo local. El planteo fue asegurar el DU como eje director en pos de sostener niveles de empleo e índices de actividad económica, y con ello fortalecer el rol del gobierno local en un contexto de creciente competitividad interurbana.

En este marco surgen las propuestas de planificación urbana estratégica, modelo enfocado en dos ejes fundamentales: el crecimiento y desarrollo urbano en tanto actividad económica, por un lado; y por el otro la participación de actores empresariales ligados al negocio urbano-inmobiliario junto a organizaciones civiles para legitimar estos procesos como participativos, representativos y abiertos. Enfoque que tuvo gran éxito en los países latinoamericanos gracias a la propaganda y difusión por parte de arquitectos y lobistas que trasladaban modelos y prácticas europeas "exitosas" (Vainer, 2002; Ferreira, 2001; Delgadillo, 2014). Se desprendía de ello la necesidad de generar atractivos para la llegada de inversiones, eficientizar los servicios e infraestructuras y disminuir las restricciones y la burocratización, referido directamente a eliminar, "modernizar" o actualizar la normativa con un claro sesgo hacia la liberalización.

\section{El modelo de DU y la planificación estratégica en la ciudad (1991-1999)}

Siguiendo la tendencia de descentralización, en Argentina se produjo un cambio constitucional en 1994. La reforma, dejó establecido bajo el artículo №123 la autonomía municipal en el 
orden institucional, político, administrativo, económico y financiero ${ }^{4}$. Este hecho se convirtió en una bisagra de la organización política del país bajo el lema de modernizar el Estado.

Acompañando la descentralización administrativa nacional, en la ciudad de Córdoba se desarrolla entre 1995 y 1999 el Plan estratégico para la ciudad (PEC), el cual genera nuevas centralidades a partir de la construcción de Centros de Participación Comunal que venían a desconcentrar la tarea administrativa municipal. El PEC toma los principios de la planificación estratégica: competitividad, productividad, marketing urbano, gestión gerencial, participación de actores con intereses en el DU (Vainer, 2002). Por ello y coincidiendo con las etapas del municipalismo en la región, se reconoce que "el gobierno local pretendió asumir un nuevo papel como promotor del desarrollo económico local, dinamizador del nivel de actividad de su ciudad y generador de condiciones que favorecieran la creación de empleo" (Marianacci, 2000:12). En el mismo sentido:

“(...) No es suficiente que el municipio se oriente sólo a la prestación de un conjunto de servicios urbanos y a la concreción de obras de infraestructura y equipamiento; su nuevo papel consiste en orientarse fuertemente a la promoción del desarrollo económico y social, a escala local" (Marianacci, 2000:13).

El plan no tuvo respaldo legal por no traducirse en ningún instrumento que pudiera ser aprobado por el poder legislativo, aunque en 1997 se institucionaliza el concepto de planificación estratégica en la carta orgánica municipal como una función propia de la gestión (Vanella et al., 2001).

Uno de los argumentos centrales para la aplicación del PEC se relacionaba a la pérdida de capacidad de la planificación tradicional, que había sido utilizada hasta la fecha, en la regulación del crecimiento urbano. En cambio, esta propuesta ofrecía una alianza estratégica con los sectores empresariales interesados, asumiendo la necesidad de contar con sus recursos para el "desarrollo" de la ciudad y lograr la ansiada inserción y competitividad en el mercado global. El plan seguía fielmente los objetivos planteados en la NAU, convertir la estructura urbana en eficiente y competitiva, atraer inversiones en el mercado global y orientar la ciudad hacia un "desarrollo sostenible".

De las líneas de intervención que se plantearon -económica, urbana, ambiental y social- la subordinación al primer eje fue evidente. En gran medida los proyectos urbanos eran de alcance sectorial con la casi exclusiva participación de actores privados relacionados a la actividad empresarial-comercial, como lo fueron la habilitación de "mega emprendimientos" comerciales tipo hipermercados y shoppings, fundamentalmente en el anillo periférico. Junto con ello, se decide no continuar con el control de la ocupación periférica sino todo lo opuesto, fomentarla a través del plan de descentralización municipal y el incentivo a la localización residencial en torno a estas nuevas centralidades. Para ello, se produjo una fuerte liberalización normativa del suelo (excepciones para el desarrollo de barrios cerrados y country) tensionando el modelo anterior (Figura 3).

Sin embargo, como no está específicamente reglada, cada provincia amplia, reduce o gestiona dicha autonomía. 
Figura No3.

Áreas de desarrollo en la ciudad de Córdoba según PEC. Córdoba, 1996

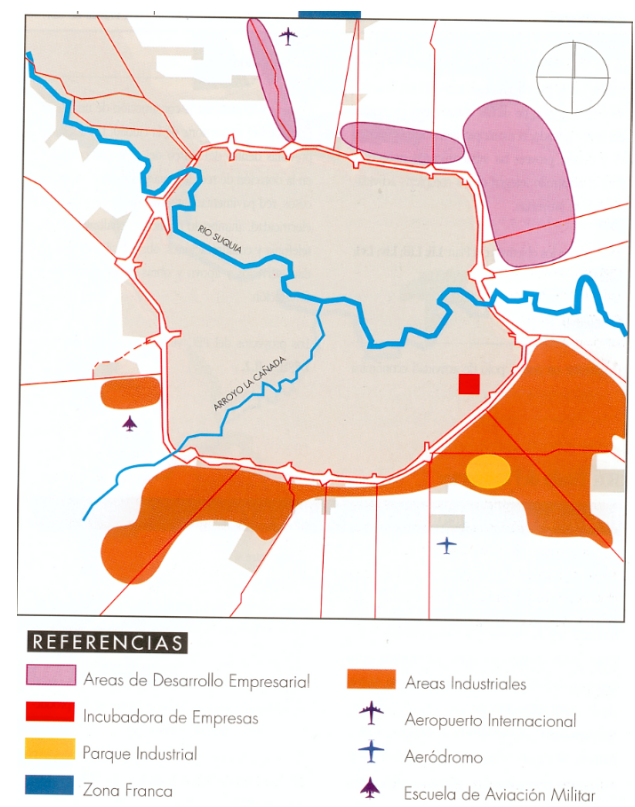

Fuente: Archivo histórico municipal, Municipalidad de la ciudad de Córdoba.

\section{El foro urbano mundial y el foco en la gestión local en los primeros 2000}

El paradigma globalizador y totalizante del modelo de planificación estratégica se topó con las particularidades y conflictos coyunturales en los intentos de implementación local que se dieron en la región. Acorde a ello, los documentos del BM para el DU se enfocaron en apoyar la escala local y promover una serie de estrategias específicas para su implementación. Entre otras se incluye la necesidad de respaldar y fortalecer los principios del DU como eje prioritario para las agendas de los gobiernos locales, concentrando las acciones y estrategias en dicha escala (Van Lindert, 2016). En este marco, se intensificó la difusión de proyectos y experiencias particulares que pretendían atender las demandas específicas de lo local, tales como: la planificación sectorial para estimular áreas degradadas; la formulación de proyectos específicos; mayor participación de actores privados como estrategia para atender las demandas de agentes locales; la reproducción de "buenas prácticas urbanas" como ejemplos pragmáticos de ejecución de proyectos, entre las más destacadas.

Así también comenzaron a circular documentos elaborados por organismos especializados acerca del funcionamiento del mercado de suelo urbano y junto con ello la noción de recuperación de plusvalor (Jaramillo, 2003; Maldonado et al., 2006) alimentando el debate y la implementación de estas ideas. Fundamentalmente se plantea que los gobiernos locales están en condiciones de otorgar una serie de condiciones particulares a determinados emprendimientos urbano-inmobiliarios con el fin de compartir un porcentaje de la ganancia potencial estimada y obtener con ello ingresos u obras para la ciudad. Esta última estrategia, logró ocuparse simultáneamente de la necesidad de difundir prácticas locales específicas junto con la internacionalización de modalidades de producción urbana como los grandes proyectos urbanos. 
Por otra parte, desde la ONU, se reforzaron los principios propuestos por la NAU, pero esta vez a través de otro formato: el Foro urbano mundial. El mismo comenzó a ejecutarse a partir de 2002 bajo la consigna de propiciar urbanizaciones sustentables. El Foro impulsa la institucionalización de los procesos participativos con todos los agentes que busquen un desarrollo y una gestión sostenible de las ciudades (Montes, 2001), incentivando con ello la legitimización de prácticas e instrumentos. Simultáneamente, desde CEPAL, se propone instalar el ordenamiento territorial como instrumento que concilia los intereses de la planificación tradicional -basada en la proyección física espacial a largo plazo- con la planificación estratégica, pensada para intervenciones puntuales o sectoriales en el mediano y corto plazo. La combinación intenta conciliar las críticas hacia la planificación estratégica, acusada de concentrar los objetivos exclusivamente hacia el desarrollo económico sin atender otras condiciones sociales relevantes del contexto, aunque valorando la flexibilidad, la eficiencia y la incorporación de actores privados que trajo como dogma, en tanto puntos claves para la gestión urbana. Los instrumentos de la planificación tradicional son considerados por estos organismos como pocos operativos, desfasados temporalmente e insuficientes para la complejidad de la problemática urbana que se encuentra en "permanente cambio y necesita herramientas flexibles" (Montes, 2001).

El ordenamiento territorial como opción de política urbana logra impacto en numerosos municipios de la región, que miraron con buenos ojos integrar las preocupaciones en torno a la sustentabilidad y el cuidado del medio ambiente con los objetivos propuestos para el DU desde la planificación estratégica. Se destaca la simultánea aprobación de planes de ordenamiento territorial en diferentes escalas gubernamentales que incluian en su contenido una serie de instrumentos de gestión. En varios países se dio también una masiva aceptación y reproducción de dichos instrumentos, argumentados como estrategias para favorecer el DU bajo los preceptos antes descriptos, aun en ausencia de planes de ordenamiento territorial. La innovación radica en que, mediante ellos, es posible alcanzar un nuevo nivel operativo, más eficiente y flexible, a la hora de implementar planes, proyectos y programas de política urbana gracias a que implican una negociación directa entre representantes estatales y privados interesados. Así, la participación tan promulgada a través del modelo de planificación estratégica adquiere un soporte institucional y con ello un mayor grado de legitimidad.

\section{La gestión urbana local como promotora (1999-2011)}

En el caso de estudio, las consecuencias del PEC se expusieron en el territorio a través de una intensa expansión de la mancha urbana, con dispar ocupación de las áreas periféricas y múltiples déficits de urbanización junto a un alto grado de fragmentación socio-económica que se hizo más evidente durante este período (Marengo et al., 2006). Sumado a ello se genera un boom constructivo-inmobiliario gracias a que la actividad se asume como refugio de valor y eje de inversión de los excedentes de rentabilidad obtenidos por los commodities agrícolas ${ }^{5}$ en un contexto de crisis de confianza en el sistema financiero-bancario; lo que dio lugar a procesos de renovación urbana en barrios peri centrales y a urbanizaciones de alto nivel adquisitivo en la periferia.

Se habla de commodities para referirse a materias primas o bienes primarios, con bajo nivel de especialización e industrialización que alcanzan valores comerciales iguales en los mercados del mundo. 
Se retoma la intención de generar un plan urbano, representado por varios documentos (Diagnóstico y líneas estratégicas para el Plan Urbano Ambiental-2001; Formulación de lineamientos alternativos para el plan urbano ambiental-2003; Plan Estratégico para la ciudad de Córdoba-PECba-2003-2006 y Bases para el Plan Director de la Ciudad de Córdoba 2008-2020), que no lograron pasar de la presentación y nunca fueron implementados.

El último documento propone una serie de novedades, siendo en principio las bases para elaborar posteriormente un Plan Director -el cual nunca se llegó a realizar- que acompaña lo planteado por el Foro urbano mundial al contener una "visión" que integra los principios de "inclusión, sustentabilidad e institucionalidad insoslayable en la gestión urbana local" (Municipalidad de la Ciudad de Córdoba, 2008:8). El último punto refiere específicamente al fortalecimiento de las capacidades institucionales, de los equipos técnicos y la necesidad de interactuar con otros sectores que contribuirían a desarrollar exitosamente los proyectos y programas propuestos, sintonizando nuevamente con las ideas del Foro. Por primera vez en la ciudad, se establecieron una serie de instrumentos concretos para los planes, programas y proyectos diferenciados según se apliquen en sectores de intervención estratégica o nodos (Figura 4). Los mismos se definieron como herramientas a utilizar por la gestión urbana, distinguiendo en acciones directas (como planes de obra pública), acciones indirectas (referido exclusivamente a cambios en el marco regulatorio) y acciones mixtas (determinando un mecanismo propio para ello: las concertaciones público-privadas).

Figura $\mathrm{N}^{\circ} 4$.

Identificación de sectores de intervención estratégica diferenciado según sean polígonos o nodos según Bases para el plan director. Córdoba, 2008.
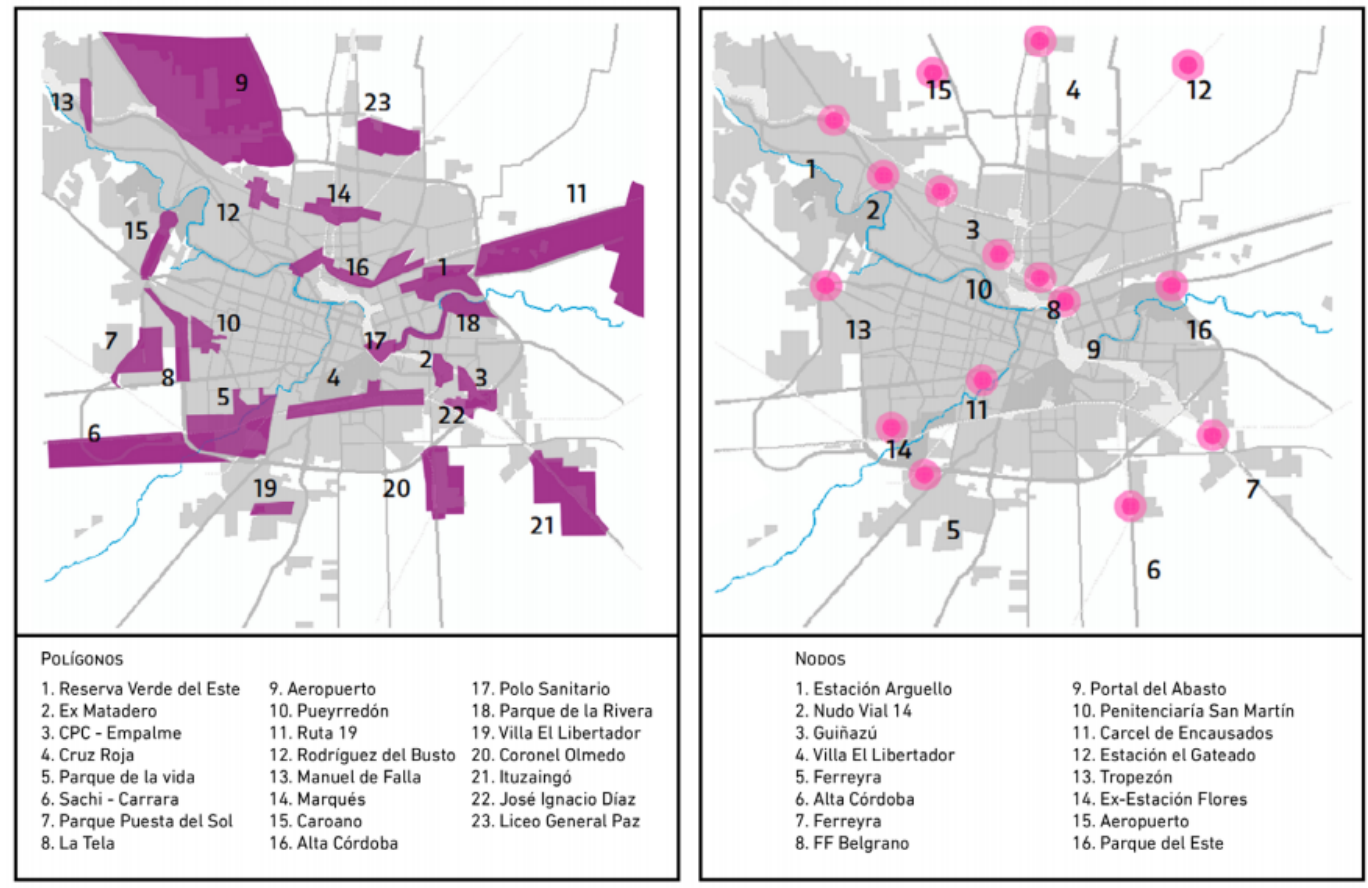

Fuente: Lineamientos y estrategia general para el reordenamiento del territorio, Municipalidad de Córdoba. 
Los múltiples intentos para consensuar un plan urbano, todos ellos sin éxito, manifiesta la paulatina separación de la planificación urbana como actividad tradicionalmente vinculada a la gestión de la ciudad. En su reemplazo, se dio cada vez más lugar a la implementación de instrumentos con eje en la recuperación de la plusvalía del suelo, en tanto mecanismo dinamizador para la producción urbana. En este sentido y durante estos años, la gestión urbana es fuertemente influenciada por las necesidades del sector empresarial urbano inmobiliario (Cisterna \& Capdevielle, 2015). Podemos decir entonces que la clave de este nuevo ciclo de gestión urbana fue asumir la capacidad de injerencia del sector empresarial en las decisiones de políticas y programas urbanos en la ciudad, dejando de lado los planes o la planificación a escala urbana para consagrar programas por sector $\mathrm{o}$ innovaciones instrumentales específicas que responden a las necesidades empresariales.

"Era necesaria una iniciativa como ésta que permitiera pasar del modelo del Estado regulador a un Estado con un rol protagónico, como promotor del desarrollo. Este modelo de administración promotora y participativa en la generación de la ciudad, sin resignar su potestad normativa, es vital a la concurrencia de los intereses públicos y privados en beneficio de la ciudad y sus vecinos, indicó el Director Ejecutivo del Grupo Edisur, Rogelio Moroni" (LaVoz, 2010).

\section{Hacia la consolidación del urbanismo neoliberal}

El documento previo al encuentro de Hábitat III, realizado por ONU Hábitat y CEPAL (2016), propone como ejes de acción para la región la necesidad de avanzar en la actualización de los marcos legales, la búsqueda de nuevas herramientas para el financiamiento, orientar la planeación urbana y territorial hacia un diseño integrador y establecer mecanismos de revisión y monitoreo.

Sobre la planificación, reconoce que desde la década del ' 80 y en un contexto de democratización de la región, se le dio énfasis al ordenamiento urbano con un largo historial de intentos por aplicar una regulación urbana compleja -como son los códigos de zonificación y planes generales- aunque han demostrado ser burocráticos y no han logrado comprender la formulación de los precios del suelo (CEPAL, 2016). El planteo actual refiere a particularizar e individualizar el alcance de las acciones proyectadas por la gestión urbana e incorporar además la participación de los agentes interesados e involucrados en las operaciones para la definición de las normas y el desarrollo de las mismas.

La mayor parte de los instrumentos implementados en estos últimos años en los gobiernos locales de la región, como así también la intensidad en su promoción por parte de algunos organismos internacionales, tratan fundamentalmente de introducir nuevas modalidades para la negociación y la articulación público-privada (Van Lindert, 2016; Delgadillo, 2014). El principal argumento se centra en la posibilidad de obtener recursos municipales y financiamiento a partir de la captación de los beneficios generados por el DU (Smolka, 2013). Los documentos sugieren a los municipios posicionarse como actores promotores que, al tener el monopolio de la potestad regulatoria, deben poder capitalizarla como un activo plausible de negociar frente a la necesidad de un mejor rendimiento y productividad de las inversiones inmobiliarias. Lo que podríamos inter- 
pretar como vehiculizar mediante estos mecanismos la lógica de competitividad y mercantilización, propia de la visión empresarial, hacia la gestión urbana.

\section{La mercantilización como eje de la gestión urbana (2011-2019)}

El último período de este análisis fue, de la misma forma que la retórica de las agencias internacionales, marcado por la profundización de las estrategias desplegadas en años anteriores. La gestión urbana asumió que el marco regulatorio que rige desde 1985 no estaba acorde a las necesidades y tiempos actuales, expresándolo a través de excepciones, negociaciones y tratamientos ad hoc. La administración municipal habilita para ello nuevos instrumentos de gestión, basadas en el aprovechamiento de la máxima capacidad constructiva y la negociación de las normas con la condición de recuperar parte del plusvalor. Durante estos años, predominó fundamentalmente la elección y utilización de los mecanismos que permiten el ingreso de recursos económicos, ya sea dinero en efectivo u obras, a través de distintos instrumentos y modalidades de concertación púbico-privada.

Apoyando este enfoque se presenta un plan de "Producción de ciudad-Áreas de promoción urbana" (Ord. No 12483, 2015) que consiste en una modificación parcial de la vieja norma de ocupación y usos del suelo para establecer "áreas de promoción" (Figura 5) en las cuales se puedan aplicar instrumentos de recuperación de plusvalor habilitados por la misma gestión anteriormente.

Figura N05.

Áreas de promoción urbana según "Modelo de producción de ciudad". Córdoba, 2015.

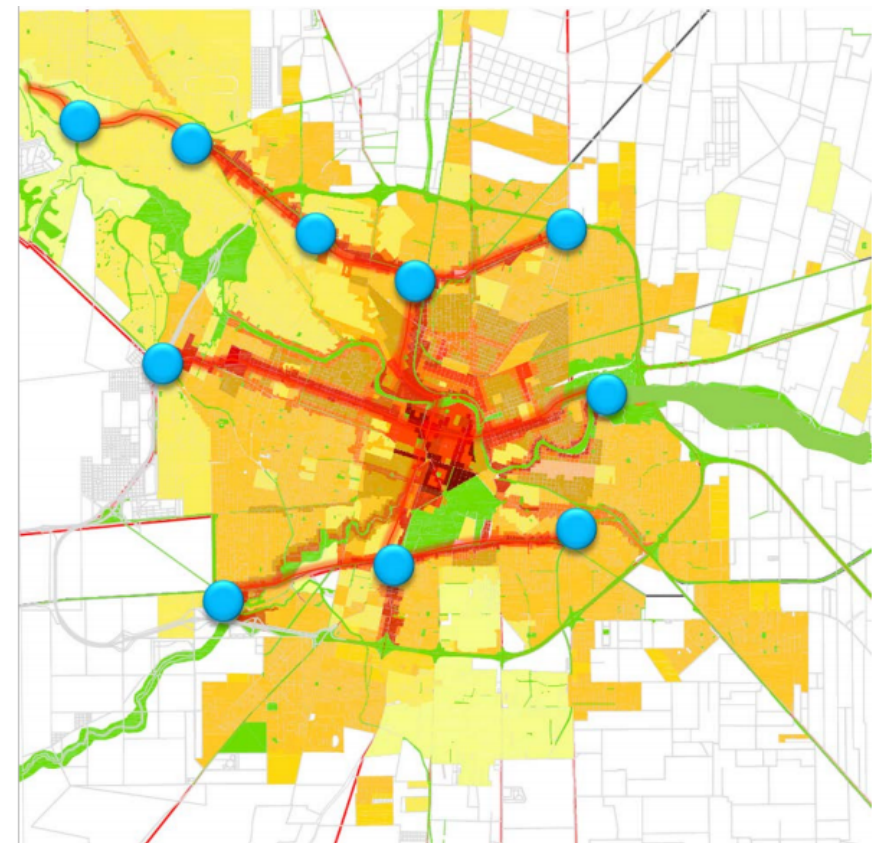

Fuente: Municipalidad de Córdoba. 


\section{Algunas reflexiones de cierre}

La periodización realizada intenta, de manera muy sintética, leer de manera articulada los paradigmas promovidos por las organizaciones internacionales y aquellas practicas aplicadas en la gestión y planificación urbana local durante las últimas décadas. Esta relación procura graficar la incidencia de los modelos promocionados a la vez que entendemos a estos paradigmas como herramientas para legitimar la orientación del desarrollo urbano en las diferentes coyunturas políticas-económicas. En este sentido se evidencia una pérdida de escala e incidencia en las políticas y en la gestión de la planificación urbana en tanto proyecto, control y limitación de lo urbano para ser suplantada por acciones puntuales que van a apuntalar y sostener el desarrollo económico-productivo apoyado en un modelo de gobernanza y participación, aunque ésta última sólo dé lugar a los intereses y actores empresariales.

La genealogía elaborada nos permitió también realizar una lectura temporal y relacional entre los paradigmas difundidos y la gestión y planificación urbana realmente existente en un caso de estudio. En los primeros años del análisis se logra identificar la influencia a nivel conceptual, aunque con algunos matices y mixturas en su aplicación por herencias en la práctica de planificación. En cambio, a partir de la década del ' 90 se manifiestan de manera inmediata y prácticamente sin mediaciones en el enfoque y las herramientas utilizadas por la gestión urbana local, configurando en su recorrido hasta la fecha una adaptación paulatina y continua de la planificación hacia modalidades mercantilizadas y neoliberales.

A modo de síntesis, el siguiente gráfico reúne los conceptos, estrategias y prácticas de territorialización expuestos.

Como señalamos al inicio, las ciudades se han convertido en los últimos años en un campo de incubación, experimentación y despliegue de programas, prácticas y modelos que efectivizan la aplicación de la agenda neoliberal. Una escala de acción que juega un papel central en las condiciones en que se desenvuelve el desarrollo urbano realmente existente. Recuperando a Janoschka (2011) se ha prestado mucho interés en las consecuencias territoriales del neoliberalismo urbano, aunque no así en conocer cuáles y cómo son los procesos que provocan esas reconfiguraciones urbanas. En este sentido, nos preguntamos qué nuevas prácticas, modelos y paradigmas trae la agenda neoliberal a la planificación y la gestión de las ciudades latinoamericanas. 
Figura $\mathrm{N}^{\circ} 6$.

Síntesis de paradigmas y modelos difundidos y de enfoques y estrategias aplicados en la gestión y planificación urbana local durante las últimas décadas.

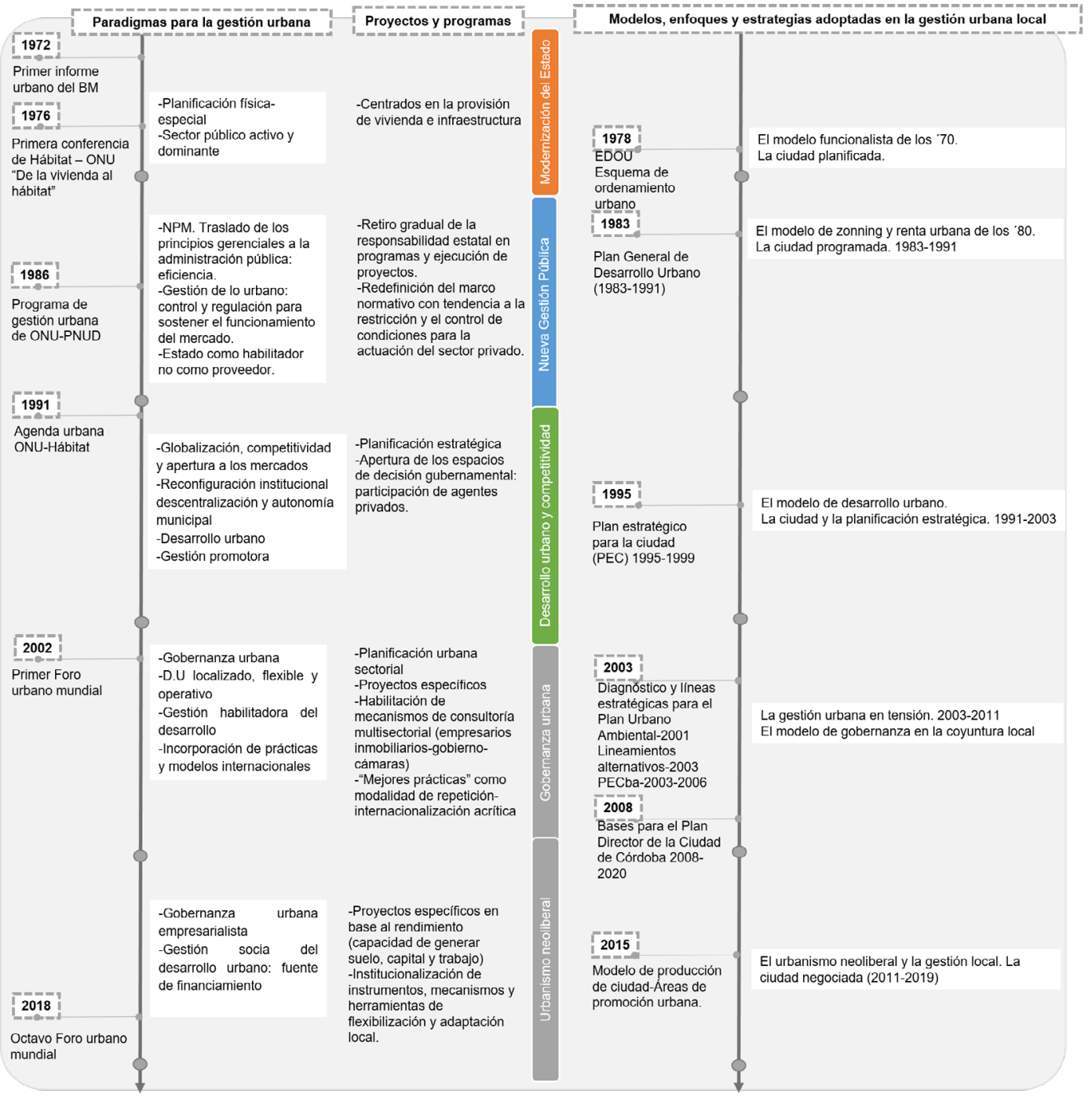

Fuente: Elaboración propia en base a documentos oficiales y fuentes bibliográficas.

\section{Bibliografía}

AGHÓN, G., ALBURQUERQUE, F. \& CORTÉS, P. Desarrollo económico local y descentralización en América Latina: Análisis comparativo. Santiago de Chile: CEPAL, 2001.

BALLENT, A. Las huellas de la política. Vivienda, ciudad, peronismo en Buenos Aires, 1943-1955. Buenos Aires, Argentina: Prometeo, 2005. 
BRENNER, N. \& THEODORE, N. Cities and the Geographies of «Actually Existing Neoliberalism». Antipode, 2002, Vol. 34, N³, p. 349-379.

CAO, H., REY, M. \& DUCA, A.L. Ajuste estructural y sociocentrismo: el discurso de la gobernanza. Administración Pública y Sociedad (APyS), 2016, º1, p. 6-20.

CAO, H. \& VACA, J. El fracaso de la descentralización argentina. Andamios, Revista de Investigación Social, 2007, Vol. 4, №7, p. 249-267.

CEPAL. Plan de Accion Regional para la implementacion de La Nueva Agenda Urbana 2016-2036. Santiago de Chile: CEPAL-Programa de las Naciones Unidas para los Asentamientos Humanos, 2016.

CAPOROSSI, C. Planificación y crecimiento urbano. Ideas y reflexiones a partir del caso de la ciudad de Córdoba. La periferia de Córdoba. Cuestiones sobre el hábitat. Córdoba: Departamento de publicaciones FAUD - UNC, 2006.

CISTERNA, C. \& CAPDEVIELLE, J. Estrategias de promocion inmobiliaria en la produccion de la ciudad. El caso del "desarrollista» GAMA en la ciudad de Cordoba, Argentina. Papeles de Geografía, 2015, Vol. 61, p. 137-153.

COLAUTTI, V. Procesos de transformación urbana en la ciudad de Córdoba. V Seminario Internacional de Investigación en Urbanismo, Barcelona-Buenos Aires. Barcelona: DUOT, 2013, p. 480-495.

DE MATTOS, C. Globalización, negocios inmobiliarios y transformación urbana. Nueva Sociedad, 2007, No212, p. 82-96.

DEL RÍO, J., VÉRTIZ, F. \& URSINO, S. La acción pública en el espacio urbano. Debates y reflexiones en torno a la noción de política urbana. Estudios Sociales Contemporáneos, 2014, Vol. 11, p. 76-86.

DELGADILLO, V. Urbanismo a la carta: teorías, políticas, programas y otras recetas urbanas para ciudades latinoamericanas. Cadernos Metrópole, 2014, Vol. 16, №31, p. 89-111.

FERREIRA, J.S.W. Governança, um novo paradigma de gestão? Sobre a Conferência de Pierre Calame. Pós. Revista do Programa de Pós-Graduação em Arquitetura e Urbanismo da FAUUSP, 2001, Vol. 9, N¹0, p. 126.

GASPAR, R.C. Estado, instituciones y desarrollo urbano. En FLACSO-MINISTERIO DE CULTURA DEL ECUADOR (Ed.). Lo urbano en su complejidad: una lectura desde América Latina. Quito, Ecuador, 2008.

HARVEY, D. Espacios del capital. Madrid: Akal, 2007.

INDEC. Censo Nacional de Población, Hogares y Viviendas, 2010. 
IROS, G. Desarrollo urbano. Reflexiones y acciones. Córdoba: Editorial Municipal de la Ciudad de Córdoba, 1991.

JANOSCHKA, M. Geografías urbanas en la era del neoliberalismo. Una conceptualización de la resistencia local a través de la participación y la ciudadanía urbana. Investigaciones Geográficas, 2011, Vol. 76, p. 118-132.

JARAMILLO, S. Los fundamentos económicos de la "participación en plusvalías". Lincoln Institute of Land Policy, 2003.

LA VOZ. En procura de una política de Estado fundada en el acuerdo y la concertación. Desarrollo Urbano. 23 de septiembre de 2010. Disponible en https://www.lavoz.com.ar/municipalidad-de-cordoba/en-procura-de-una-politica-de-estado-fundada-en-el-acuerdo-y-la-concertacion

LÓPEZ, A. Los fundamentos de la Nueva Gestión Pública: lógica privada y poder tecnocrático en el Estado mínimo. En M.Thwaites \& A. López (Ed.). Entre tecnócratas globalizados y políticos clientelistas. Derrotero del ajuste neoliberal en el Estado argentino. Buenos Aires: Prometeo, 2005, p. 1-13.

MALDONADO, M., PINILLA, F., RODRÍGUEZ, F. \& VALENCIA, N. Planes parciales, gestión asociada y mecanismos de distribución equitativa de cargas y beneficios en el sistema urbanístico colombiano. Lincoln Institute of Land Policy, 2006.

MALECKI, J.S. Ernesto La Padula en Córdoba: peronismo y ciudad, 1946-1955. Anuario de Estudios Americanos, 2018, Vol. 75, Nº1, p. 323.

MARENGO, C., DÍAZ TERRENO, F., PERALTA, C., FORNÉ, M., OCHOA, A., CAPOROSSI, C., LIBORIO, M. \& MONAYAR, V. La Periferia de Córdoba: cuestiones sobre hábitat urbano. Córdoba: Departamento de publicaciones FAUD - UNC, 2006.

MARIANACCI, G. Descentralización y desarrollo económico local: el estudio de caso de la ciudad de Córdoba, Argentina. Santiago de Chile: CEPAL, 2000.

MONTES, P. El ordenamiento territorial como opción de políticas urbanas y regionales en América Latina y el Caribe. Santiago de Chile: CEPAL, 2001.

MUNICIPALIDAD DE LA CIUDAD DE CÓRDOBA. Plan Estratégico para la Ciudad, 1996.

MUNICIPALIDAD DE LA CIUDAD DE CÓRDOBA. Bases para el Plan Director de la Ciudad de Córdoba. Lineamientos y Estrategia General para el reordenamiento del territorio, 2008.

PECK, J., THEODORE, N. \& BRENNER, N. Neoliberal urbanism: models, moments, mutations. SAIS Review, 2009, Vol. 29, No1, p. 49-66.

PRADILLA COBOS, E. Las políticas neoliberales y la cuestión territorial. Cuadernos de Economía, 1990, Vol. 10, No14, p. 1-19. 
PRADILLA COBOS, E. Las politicas y la planeacion urbana en el neoliberalismo. En P. Brand (Comp.). La ciudad latinoamericana en el siglo XXI. Globalización, neoliberalismo, planeación. Medellín: Escuela de Planeación Urbana y Regional, Universidad Nacional de Colombia, 2009, p.287-308.

PRADILLA COBOS, E. Empresarios, gobiernos y ciudadanos en la disputa por la ciudad. En A. Ribeiro,T. Egler \& F. Sánchez (Comps). Política governamental e ação social no espaço. Rio de Janeiro: ANPUR-Letra Capital, 2012, p. 23-38.

PUEBLA CADENA, C. Del intervencionismo estatal a las estrategias facilitadoras : los cambios en la política de vivienda en México, (1972-1994). México, D.F.: El Colegio de México, Centro de Estudios Demográficos y de Desarrollo Urbano, 2002.

SMOLKA, M., 2013. Implementación de la Recuperación de Plusvalías en América Latina. Políticas e Instrumentos para el Desarrollo Urbano: Lincoln Institute of Land Policy.

STANG, J.I. El urbanismo como nueva disciplina en Argentina visto desde el especialista Benito J. Carrasco a través del Plan Regulador y de Extensión para la ciudad de Córdoba, 1927. Cuaderno Urbano, 2017, Vol. 23, p. 169-197.

TECCO, C. \& VALDÉS, E. Segregación residencial socioeconómica (SRS) e intervenciones para contrarrestar sus efectos negativos: Reflexiones a partir de un estudio en la ciudad de Córdoba, Argentina. Cuadernos de Geografía, 2006, №15, p. 53-66.

VAINER, C. Pátria, empresa e mercadoria. A cidade do pensamento único. Desmanchando consensos. Petrópolis RJ- Brasil: Ed. Vozes, 2002.

VANELLA, R., LUCCA, C., PITTARI, J.R., STEINBERG, F., ZWANENBURG, M. y CRUZ, S. El Plan Estratégico y el Desarrollo Económico Local de la Ciudad de Córdoba, Argentina. Santa Cruz de la Sierra: IHS-SINPA, 2001.

VAN LINDERT, P. Rethinking urban development in Latin America: A review of changing paradigms and policies. Habitat International, 2016, Vol. 54, p. 253-264.

ZANETTA, C. The evolution of the World Bank's urban lending in Latin America: From sites and services to municipal reform and beyond. Habitat International, 2001, Vol. 24, N4, p. 513-533. 